\title{
Noradrenergic Synaptic Function in the Bed Nucleus of the Stria Terminalis Varies in Animal Models of Anxiety and Addiction
}

\author{
Zoé A McElligott' ${ }^{1,2}$, Megan E Fox', Paul L Walsh', Daniel J Urban ${ }^{3}$, Martilias S Ferrel $^{3}$, Bryan L Roth ${ }^{3}$ and \\ R Mark Wightman*,1,2,4
}

'Department of Chemistry, University of North Carolina at Chapel Hill, Chapel Hill, NC, USA; ${ }^{2}$ Bowles Center for Alcohol Studies, University of North Carolina at Chapel Hill, Chapel Hill, NC, USA; ${ }^{3}$ Department of Pharmacology, University of North Carolina at Chapel Hill, Chapel Hill, NC, USA; ${ }^{4}$ Center for Neuroscience, University of North Carolina at Chapel Hill, Chapel Hill, NC, USA

\begin{abstract}
Lewis rats show increased anxiety-like behaviors and drug consumption compared with Sprague-Dawley rats. Prior work suggests norepinephrine (NE) signaling in the bed nucleus of the stria terminalis (BNST) could have a role in mediating these phenotypes. Here, we investigated NE content and dynamics in the ventral BNST (vBNST) using fast-scan cyclic voltammetry in these two rat strains. We found that NE release evoked by electrical stimulus and its subsequent uptake was dysregulated in the more anxious Lewis rats. Because addiction is a multifaceted disease influenced by both genetic and environmental factors, we hypothesized NE dynamics would vary in these strains after the induction of a physical dependence on morphine. Following naloxone-precipitated morphine withdrawal, NE release and uptake dynamics were not changed in Lewis rats but were significantly altered in Sprague-Dawley rats. The alterations in Sprague-Dawley rats were accompanied by an increase in anxiety-like behavior in those animals as measured with the elevated plus maze. These studies suggest novel mechanisms involved in the development of affective disorders, and highlight the noradrenergic system in the vBNST as a common substrate for the manifestation of pathological anxiety and addiction.

Neuropsychopharmacology (2013) 38, I665-1673; doi:I0.1038/npp.2013.63; published online 3 April 2013
\end{abstract}

Keywords: Norepinephrine; bed nucleus of the stria terminalis; morphine; neuronal release; norepinephrine uptake

\section{INTRODUCTION}

In drug-dependent individuals, researchers have highlighted the development of a persistent 'negative emotional state' when access to drugs is terminated (Koob, 2009). Substance abuse is often co-morbid with anxiety disorders and impacted by stressful life experiences (Hyman et al, 2009; Sinha, 2008). The risk for developing an addiction, however, varies considerably between individuals, and different people can have very different responses to drug or stress exposure. One means for addressing this issue is examining animals with divergent behavioral phenotypes in appropriate neuronal structures. Akin to human addicts, inbred Lewis (L) rats self-administer opiates at high levels (George and Goldberg, 1989), and show escalation of drug-taking behavior (Picetti et al, 2012). Additionally, L rats display several anxiety-like phenotypes as compared with outbred Sprague-Dawley (SD) rats, as well as hypocortisolemia; thus,

\footnotetext{
*Correspondence: Dr RM Wightman, Department of Chemistry, University of North Carolina at Chapel Hill, Campus box \#3290, Chapel Hill, NC 27599-3290, USA, Tel: +919 962 |472, Fax: +919 962 2388, E-mail: rmw@unc.edu

Received 4 October 2012; revised 28 February 2013; accepted 4 March 2013; accepted article preview online 6 March 2013
}

they have been suggested to be a good model of posttraumatic stress disorder (PTSD) (Cohen et al, 2006).

The bed nucleus of the stria terminalis (BNST) is a forebrain nucleus in the extended amygdala positioned to relay between cortical, hippocampal and amygdalar inputs, and stress and reward centers (Drolet, 2009). The BNST is densely innervated by noradrenergic fibers arising from the $\mathrm{A} 1, \mathrm{~A} 2$ (via the ventral noradrenergic bundle or VNAB), and A6 (via the dorsal noradrenergic bundle) cell bodies (Forray and Gysling, 2004). NE signaling within the BNST modulates anxiety-like behavior and influences induction of the hypothalamic-pituitary-adrenal (HPA) axis (Cecchi et al, 2002), affects expression of learned and physical opiate withdrawal behaviors (Delfs et al, 2000) and contributes to stress-induced reinstatement of drug seeking (Erb et al, 2000; Leri et al, 2002; Wang et al, 2001). Furthermore, NE impacts both excitatory (McElligott and Winder, 2009) and inhibitory synaptic transmission (Dumont and Williams, 2004), induces synaptic plasticity (McElligott and Winder, 2008), and releases corticotropinreleasing factor (CRF) in the BNST (McElligott et al, 2010; Nobis et al, 2011). The Morilak lab found that extracellular NE in the BNST was the same in non-manipulated L and SD rats; however, when restrained for $30 \mathrm{~min}$, extracellular $\mathrm{NE}$ 
was elevated in $\mathrm{L}$ rats compared with $\mathrm{SD}$ rats (Pardon et al, 2002). Furthermore, $\mathrm{L}$ rats exhibit fear generalization and anxiety-like behaviors that are BNST dependent (Duvarci et al, 2009).

Here, we hypothesized that the noradrenergic system projecting to the BNST may be differently regulated in these two rat strains. Furthermore, we tested whether the noradrenergic system is differentially modulated in a model of morphine addiction. Previously, we demonstrated that $\mathrm{NE}$ dynamics and their regulation can be examined in the BNST with fast-scan cyclic voltammetry (Herr et al, 2012; Park et al, 2009). We used this technique in the ventral BNST (vBNST) to investigate the mechanisms underlying differences in NE overflow in L rats as compared with SD rats, and to test the hypothesis that morphine dependence alters regulatory mechanisms at noradrenergic neurons. We found that non-manipulated $\mathrm{L}$ rats had a reduced rate of clearance and decreased sensitivity at their $\alpha_{2}$-adrenergic receptors (ARs) as compared with SD rats. Furthermore, using high-performance liquid chromatography (HPLC), we found that non-manipulated $\mathrm{L}$ rats had higher NE tissue content. When SD rats were made physically dependent on morphine (Schulteis et al, 1999), modeling the condition of a human addict, there was a significant reduction in their NE uptake rate and in their response to a challenge with an $\alpha_{2}$-AR antagonist as compared with controls. In contrast, neither clearance rate nor $\alpha_{2}$-AR sensitivity were different in morphine-dependent $\mathrm{L}$ rats as compared with control or non-manipulated animals. Correlated to these alterations at NE synapses in the BNST, morphine-dependent SD rats, but not L rats, showed heightened anxiety-like behavior as compared with controls. The changes that occurred in morphine-dependent SD rats were profound: they exhibited indistinguishable NE uptake rates and similar responses to an $\alpha_{2}$-AR antagonist as non-manipulated L rats. Thus, noradrenergic synapses underwent a remarkable adaptation when SD rats were made physically dependent on morphine. Furthermore, the data revealed that the vBNST $\mathrm{NE}$ system of the non-manipulated $\mathrm{L}$ rat resembled a 'morphine-dependent state'.

\section{MATERIALS AND METHODS}

\section{Animals Care}

All experiments were performed in accordance with the University of North Carolina at Chapel Hill (UNC) Institutional Animal Care and Use Committee's guidelines. $\mathrm{SD}$ and $\mathrm{L}$ rats (males; $350-450 \mathrm{~g}$; L rats total $n=70$, SD total $n=85$; Charles River, Wilmington, MA) were housed within UNC animal facilities and given food and water ad libitum. Electrochemical, biochemical, and behavioral experiments were performed as follows: voltammetry, SD $n=34$, L $n=26$; HPLC, SD $n=22$, L $n=15$; autoradiography, $\mathrm{SD} n=14, \mathrm{~L} n=13$; elevated plus maze, $\mathrm{SD} n=15, \mathrm{~L} n=16$.

\section{Evoked NE Release}

NE release in the vBNST was evoked from stimulation of the VNAB as previously described (Park et al, 2009) (Supplementary Methods).
Determination of NE and Dopamine Content in Tissue Slices

Brains were rapidly removed from anesthetized rats (urethane $1.5 \mathrm{mg} / \mathrm{kg}$ ). Coronal sections (300 $\mu \mathrm{m}$ thick) were prepared using a Lancer Vibratome (World Precision Instruments, Sarasota, FL) in $4{ }^{\circ} \mathrm{C}$ artificial cerebral spinal fluid (aCSF). The aCSF (in mM: $126 \mathrm{NaCl}, 25 \mathrm{NaHCO}_{3}, 2.45$ $\mathrm{KCl}, 12 \mathrm{NaH}_{2} \mathrm{PO}_{4}, 1.2 \mathrm{MgCl}_{2}, 2.4 \mathrm{CaCl}_{2}, 20$ HEPES, and 11 glucose) was adjusted to $\mathrm{pH} 7.4$ and oxygenated $\left(95 \% \mathrm{O}_{2} / 5 \% \mathrm{CO}_{2}\right)$. Tissue containing the vBNST or nucleus accumbens (NAc) was excised with a 1-mm punch, and homogenized in $0.1 \mathrm{~N} \mathrm{HClO}_{4}$ containing $1 \mu \mathrm{M}$ hydroquinone. Tissue processing and HPLC were performed as previously described (Park et al, 2009) (Supplementary Methods).

\section{Acute Morphine Dependence}

The morphine-dependence protocol was modified from Schulteis et al, 1999 (Supplementary Methods). Briefly, over the course of 3 days rats were injected once daily with $10 \mathrm{mg} / \mathrm{kg}$ morphine s.c., followed $4 \mathrm{~h}$ later by $1 \mathrm{mg} / \mathrm{kg}$ naloxone s.c. Behavioral, electrochemical, and autoradiography experiments were performed on the fourth day.

\section{Elevated Plus Maze}

The elevated plus maze (EPM) was used to assay anxietylike behavior. Animals were isolated in a novel cage for $5 \mathrm{~min}$ before running the maze. Animals explored the maze for $5 \mathrm{~min}$ and their movement and time spent in each section (open arms, center, enclosed arms) was measured and recorded using Ethovision software (Noldus, Netherlands).

\section{Chemicals and Drugs}

All chemicals and drugs were purchased from SigmaAldrich (St Louis, MO), with the exception of naloxone (Tocris Bioscience, Ellisville, MO), and used as received. Drugs were dissolved in sterile saline $(0.9 \%)$.

\section{Statistics}

Results are average values \pm SEM. Mainly unpaired Student's $t$-tests and 2-way analysis of variance (ANOVA) with post-hoc Bonferroni tests were used to determine statistical significance. Morphine withdrawal scores, prewithdrawal weight, and fecal boli weight were compared using a 3-way ANOVA with a post-hoc Tukey's Honestly Significant Difference (HSD) Test. Linear regression analysis was used to examine the relationship between stimulation intensity and $[\mathrm{NE}]_{\max }$. Differences were considered significant when ${ }^{*} P<0.05,{ }^{* *} P<0.01,{ }^{* *} P<0.0001$.

\section{RESULTS}

\section{Rats had Greater NE Tissue Content}

Owing to the phenotypes discussed above, we investigated the catecholamine tissue content in the nucleus accumbens (NAc) and the vBNST in L rats compared with SD rats. 
We did not see significant differences between the strains in the levels of DA in the NAc (SD: $5.25 \pm 0.96 \mu \mathrm{g} / \mathrm{g} n=5$, $\mathrm{L}: 6.06 \pm 0.32 \mu \mathrm{g} / \mathrm{g} n=5)$; however, in the vBNST, L rats had significantly more DA and NE tissue content (SD NE: $4.55 \pm 0.84 \mu \mathrm{g} / \mathrm{g} n=5$ vs L NE: $10.9 \pm 1.52 \mu \mathrm{g} / \mathrm{g} n=5$; SD DA: $1.06 \pm 0.32$ vs $3.15 \pm 0.46 n=5$; Figure $1 \mathrm{a}-\mathrm{c}$ ). We presumed the DA in the vBNST was mainly localized in NE neurons because DA is the metabolic precursor to NE, and dopaminergic projections are mainly to the dorsal lateral BNST (Park et al, 2012; Meloni et al, 2006).

\section{Rats had Altered Noradrenergic Neurotransmission Compared with SD Rats}

Next, we examined regulation of noradrenergic synaptic function using electrically-evoked release measured with fast-scan cyclic voltammetry in anesthetized rats. This method has been used extensively to characterize regulation of DA and serotonin neurotransmission (John et al, 2006), and we recently demonstrated its use to measure NE dynamics in vivo in the vBNST (Herr et al, 2012; Park et al, 2009). With the carbon-fiber microelectrode positioned directly beneath the anterior commissure, NE release was evoked by electrical stimulation of the VNAB (example electrode placement shown in Figure 1d). NE release (shown in color plots encoding voltammetric recordings over a 15-s interval) occurred upon stimulation, and its concentration fell to its original value after stimulation. Consistent with our previous report (Park et al, 2009), NE evoked release was unaffected by raclopride $(2 \mathrm{mg} / \mathrm{kg})$, a D2 receptor antagonist. In contrast, idazoxan ( $5 \mathrm{mg} / \mathrm{kg}$, IDA), an $\alpha_{2}-\mathrm{AR}$ antagonist, increased $\mathrm{NE}$ evoked release (examples in Figure 1f).
We compared stimulated release of NE in the vBNST in $\mathrm{L}$ and SD rats. The maximal amplitude of stimulated release $\left([\mathrm{NE}]_{\max }\right)$ was evaluated as a function of pulse number in the stimulus train $($ at $60 \mathrm{~Hz})$ and fit with a linear regression. Both strains responded to stimulation linearly, although the slope of the line was greater in the $\mathrm{L}$ rats ( $\mathrm{L}$ vs $\mathrm{SD}$, respectively, $r^{2}=0.98$ vs 0.99 , slope: $4.93 \pm 0.11$ vs $3.11 \pm 0.21, P<0.05 ;$ Figure $2 \mathrm{~b})$. The clearance half-life $\left(t_{1 / 2}\right.$ or time from the $[\mathrm{NE}]_{\max }$ to half the maximal concentration) following $60 \mathrm{~Hz} 60$ pulse stimulations was significantly greater in $\mathrm{L}$ rats than in $\mathrm{SD}$ rats $(2.6 \pm 0.2 \mathrm{~s}$, $n=6$ vs $1.9 \pm 0.1 \mathrm{~s}, n=11$ respectively, $P<0.05$; Figure $2 \mathrm{c}$ ). Additionally, when a saturating dose of the $\alpha_{2}$-AR antagonist IDA ( $5 \mathrm{mg} / \mathrm{kg}$ i.p.; see Figure 3 for dose response curve) was administered under the same stimulation conditions to $\mathrm{L}$ rats, the increase in $[\mathrm{NE}]_{\max }$ was significantly reduced compared with SD rats $(163 \pm 11 \%$ of pre-drug baseline, $n=8$ vs $217 \pm 14 \%$ of pre-drug baseline $n=10$, respectively, $P<0.05$; Figure $3 c$ ), further suggesting that NE dynamics are different in these strains. Autoradiography for $\alpha_{2}$-AR and the norepinephrine transporter (NET), however, showed no differences between the strains (SD $n=4, \mathrm{~L} n=4$, Supplementary Figure 1),suggesting total protein levels are the same.

Following this characterization, we probed the effect of an acute morphine injection ( $5 \mathrm{mg} / \mathrm{kg}$ i.p.) on NE dynamics. Surprisingly, the acute morphine slightly but significantly increased $[\mathrm{NE}]_{\max }$ in $\mathrm{L}$ rats but not in SD rats $(116 \pm 9 \%$ of pre-drug baseline, $n=5, P<0.05$ vs $91 \pm 5 \%$ of pre-drug baseline, $n=5$; Figure $3 \mathrm{e}$ ). There was no change in $t_{1 / 2}$ in either strain following acute morphine (L rats: $118 \pm 15 \%$ of pre-drug baseline, $n=5$; SD rats: $120 \pm 12 \%$ of pre-drug baseline, $n=9$; Figure 3f). Furthermore, when the response
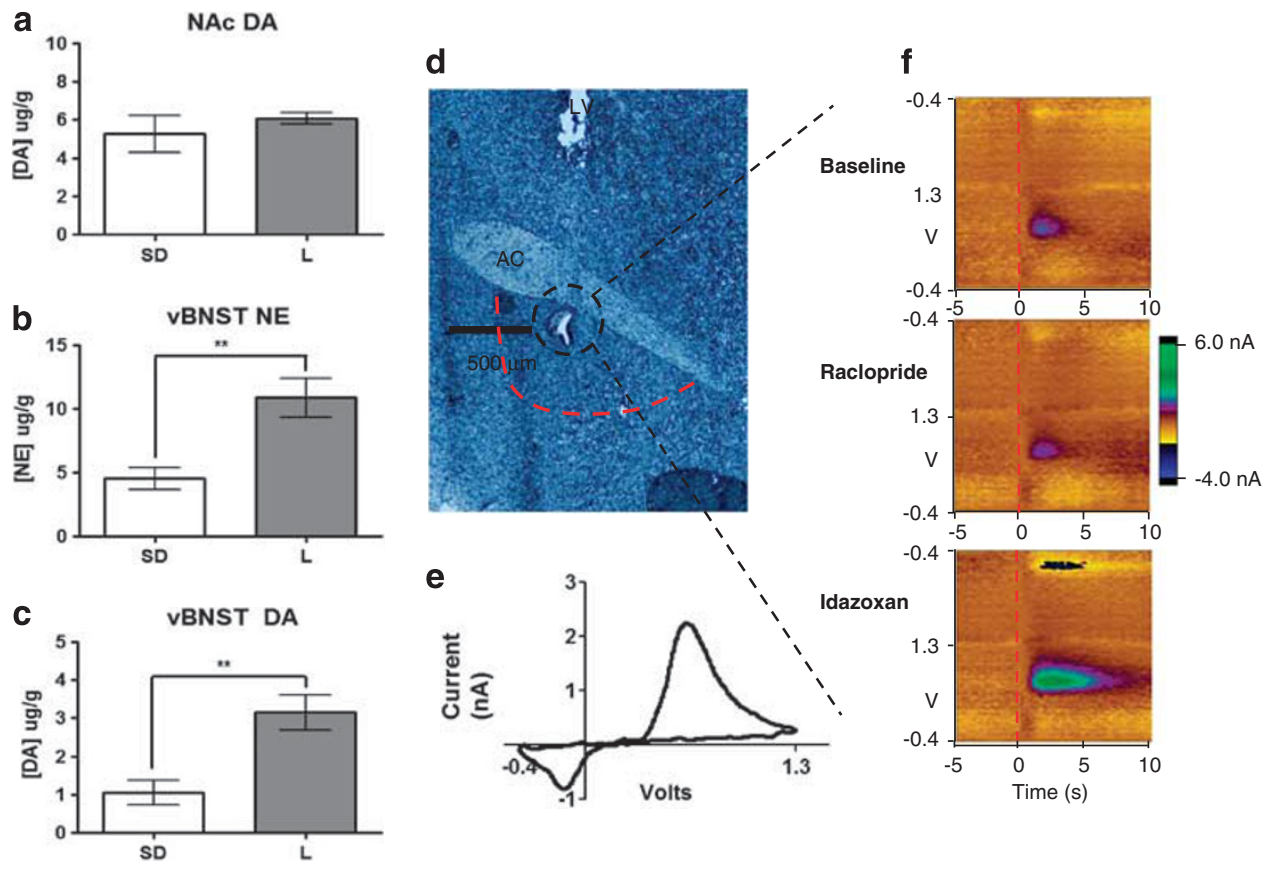

Figure I Fast-Scan Cyclic Voltammetry of norepinephrine (NE) in the bed nucleus of the stria terminalis (BNST). (a-c) Tissue content analysis of catecholamines in the BNST and nucleus accumbens (NAc). (d) Representative histology of recording electrode placement in the ventral BNST (vBNST) (black dashed circle) and area excised for tissue content analysis (red dashed line; LV, lateral ventricle; AC, anterior commissure). (e) Representative cyclic voltammogram of $\mathrm{NE}$ at $[\mathrm{NE}]_{\max }$. (f) Representative color plots demonstrating NE release and uptake in baseline conditions, following $2 \mathrm{mg} / \mathrm{kg}$ raclopride, or $5 \mathrm{mg} / \mathrm{kg}$ idazoxan. 
a
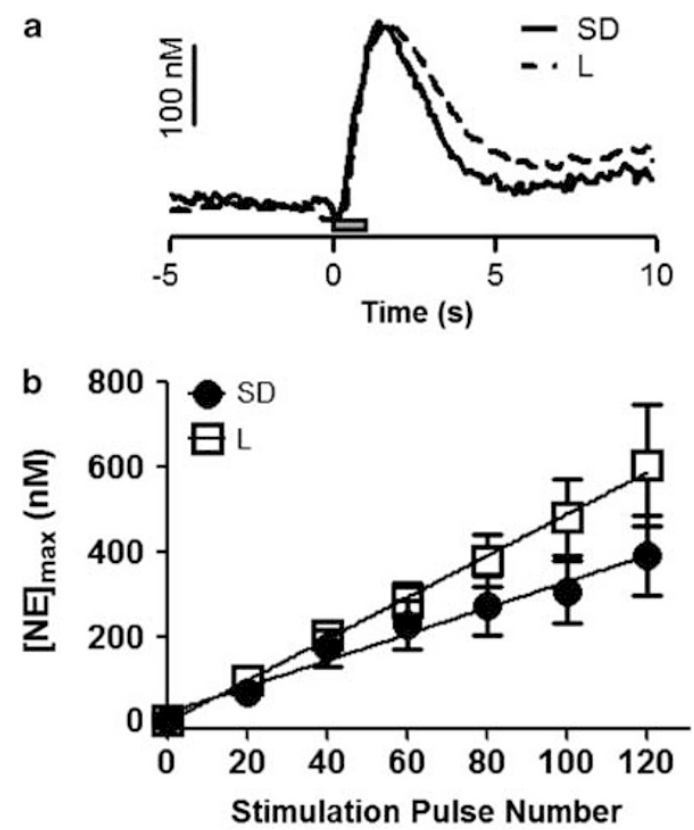

C

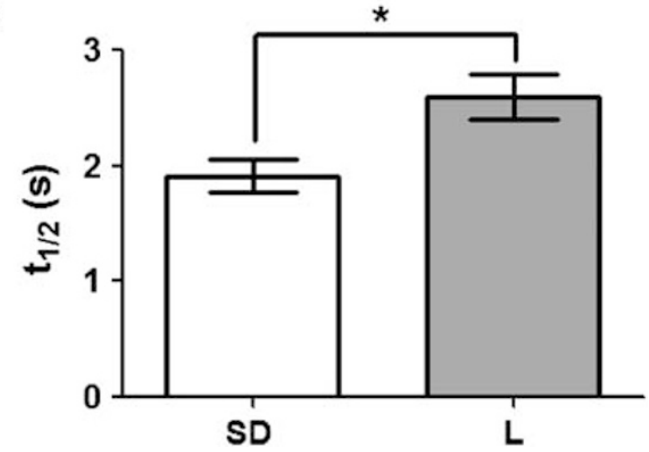

Figure 2 Comparison of norepinephrine (NE) dynamics in naïve Lewis $(\mathrm{L})$ and Sprague-Dawley (SD) rats. (a) Representative concentration traces demonstrating NE release and uptake in the ventral bed nucleus of the stria terminalis (VBNST) of both SD and $\mathrm{L}$ rats (scale bar, $100 \mathrm{nM}$; gray box = stimulation, 60 pulses, $60 \mathrm{~Hz}$ ). (b) Input-output curve of [NE] $\max$ at 20, $40,60,80,100$ and 120 pulses in SD and L rats. (c) Histogram of mean clearance half-life of $\mathrm{NE}$ following $60 \mathrm{~Hz}, 60$ pulse stimulation in both SD and $L$ rats.

to IDA was compared in acutely treated and nonmanipulated animals, there was a main effect of strain (2-way ANOVA, $F=9.41, P<0.01$; SD rats: $191 \pm 12.8 \%$ vs $217 \pm 14 \%$ of pre-drug baseline, $n=5$ and 10 ,with and without morphine, respectively; $\mathrm{L}$ rats: $158.6 \pm 5.4 \%$ vs $163 \pm 11 \%$ of pre-drug baseline, $n=5$ and 8 , with and without morphine, respectively ) but not of drug treatment. Therefore, acute morphine alone did not alter IDA response in either strain.

\section{Morphine-Dependent SD Rats had Increased Anxiety-like Behavior}

Next, we assayed animals that had experienced morphine withdrawal using a dependence paradigm that modeled the negative affect experienced by human addicts (Amitai et al, 2006; Koob, 2009; Schulteis et al, 1999). Once daily, we administered $10 \mathrm{mg} / \mathrm{kg}$ morphine s.c., followed by $1 \mathrm{mg} / \mathrm{kg}$ naloxone s.c. $4 \mathrm{~h}$ later (denoted $\mathrm{MN}$ ), for a total of 3 days
(SD $n=27, \mathrm{~L} n=22$ ). Control animals received a saline injection s.c. followed by $1 \mathrm{mg} / \mathrm{kg}$ naloxone $4 \mathrm{~h}$ later (denoted SN) once daily for a total of 3 days (SD $n=26$, $\mathrm{L} n=23)$. For $10 \mathrm{~min}$ following the injection of naloxone, animals were assayed for withdrawal behaviors (Supplementary Methods) and assigned a global withdrawal score (Figure 4a). There was a significant effect of drug and day on the global withdrawal score, but not of strain (3-way ANOVA, $F=25.34, P<0.0001$; Figure $4 \mathrm{~b})$. Thus, $\mathrm{SD}$ and $\mathrm{L}$ rats that received morphine had significantly greater withdrawal symptoms than their saline control counterparts. By the third day, both strains that received morphine showed withdrawal scores that were significantly different from the first day, while saline controls did not (Tukey's HSD Test, $P<0.0001)$. Furthermore, there was an effect of day and treatment, but not strain, with regard to prewithdrawal body weight $(F=15.99, P<0.001)$. Both strains that received morphine had significant decreases in prewithdrawal body weight (day 3 vs day 1, Tukey's HSD Test, $P<0.01 \mathrm{SD}, P<0.05 \mathrm{~L}$; Figure $4 \mathrm{c}$ ). As a quantitative measure of withdrawal-induced gut motility, we weighed the fecal boli produced during the $60 \mathrm{~min}$ following naloxone administration. Again, we found a significant effect of treatment but not strain $(F=59.08, P<0.0001)$ and a significant increase in day 3 s day 1 fecal boli weight (Tukey's HSD Test, $P<0.001 \mathrm{SD}, P<0.01 \mathrm{~L}$; Figure $4 \mathrm{~d}$ ). The similarity in responses to the morphine/naloxone challenge shows that morphine dependence was induced in both strains. Owing to the short half-lives of morphine and naloxone (Trescot et al, 2008), both drugs were eliminated by experimentation time on day 4 .

We next examined MN and SN animals of both strains on the EPM. Both center and enclosed arm time showed an interaction (2-way ANOVA, center: $F=5.78, \quad P<0.05$; enclosed: $F=5.49, P<0.05)$ and a main effect of strain (2-way ANOVA, center: $F=15.2, P<0.001$; enclosed: $F=14.0, P<0.001)$. Post-hoc analysis revealed significant differences between SD-MN and SD-SN time spent in the center $(62 \pm 11 \mathrm{~s}$ vs $106 \pm 6 \mathrm{~s}, n=8$ and 7 , respectively, $P<0.01)$ and the enclosed arms $(222 \pm 15 \mathrm{~s}$ vs $175 \pm 9 \mathrm{~s}$ respectively, $P<0.05)$. L-MN rats, however, failed to show any differences in time spent in the center or enclosed arms when compared with L-SN rats (center: $47 \pm 12 \mathrm{~s} v s 44 \pm 8 \mathrm{~s}$, enclosed: $239 \pm 13 \mathrm{~s}$ vs $250 \pm 11 \mathrm{~s}$, respectively, $n=8$; Figure $4 \mathrm{e}-\mathrm{g})$. No significant differences were noted in number of entries or open arm time between groups.

\section{NE Dynamics were Altered in Morphine-Dependent SD Rats but not L Rats}

After establishing morphine dependence, animals were anesthetized on day 4 and NE overflow was measured in the vBNST using fast-scan cyclic voltammetry. We found that the $t_{1 / 2}$ in SD-MN rats was significantly longer than in $\mathrm{SD}-\mathrm{SN}$ rats $(2.6 \pm 0.4 \mathrm{~s}$ vs $1.6 \pm 0.2 \mathrm{~s}, n=5$ and 6 , respectively, $P<0.05$; Figure 5a). Additionally the increase in $[\mathrm{NE}]_{\max }$, following IDA was significantly blunted in the SD-MN rats as compared with SD-SN rats $(147 \pm 9 \%$ vs $186 \pm 8 \%, n=5$ and 6 , respectively, $P<0.05$; Figure $5 \mathrm{~b}$ ). Interestingly, neither $t_{1 / 2}$ nor the response to IDA was altered by morphine dependence in the $\mathrm{L}$ rats $\left(t_{1 / 2}: \mathrm{L}-\mathrm{MN}\right.$ $2.3 \pm 0.3 \mathrm{~s}$ vs L-SN $2.6 \pm 0.3 \mathrm{~s}$, IDA: L-MN $163 \pm 25 \%$ of 


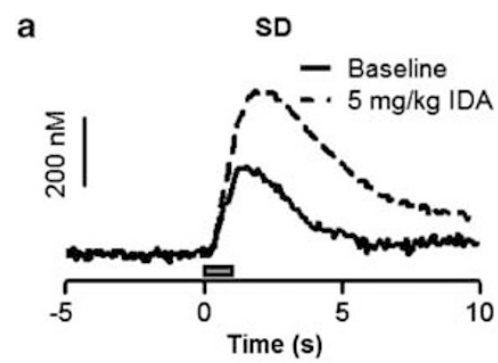

b
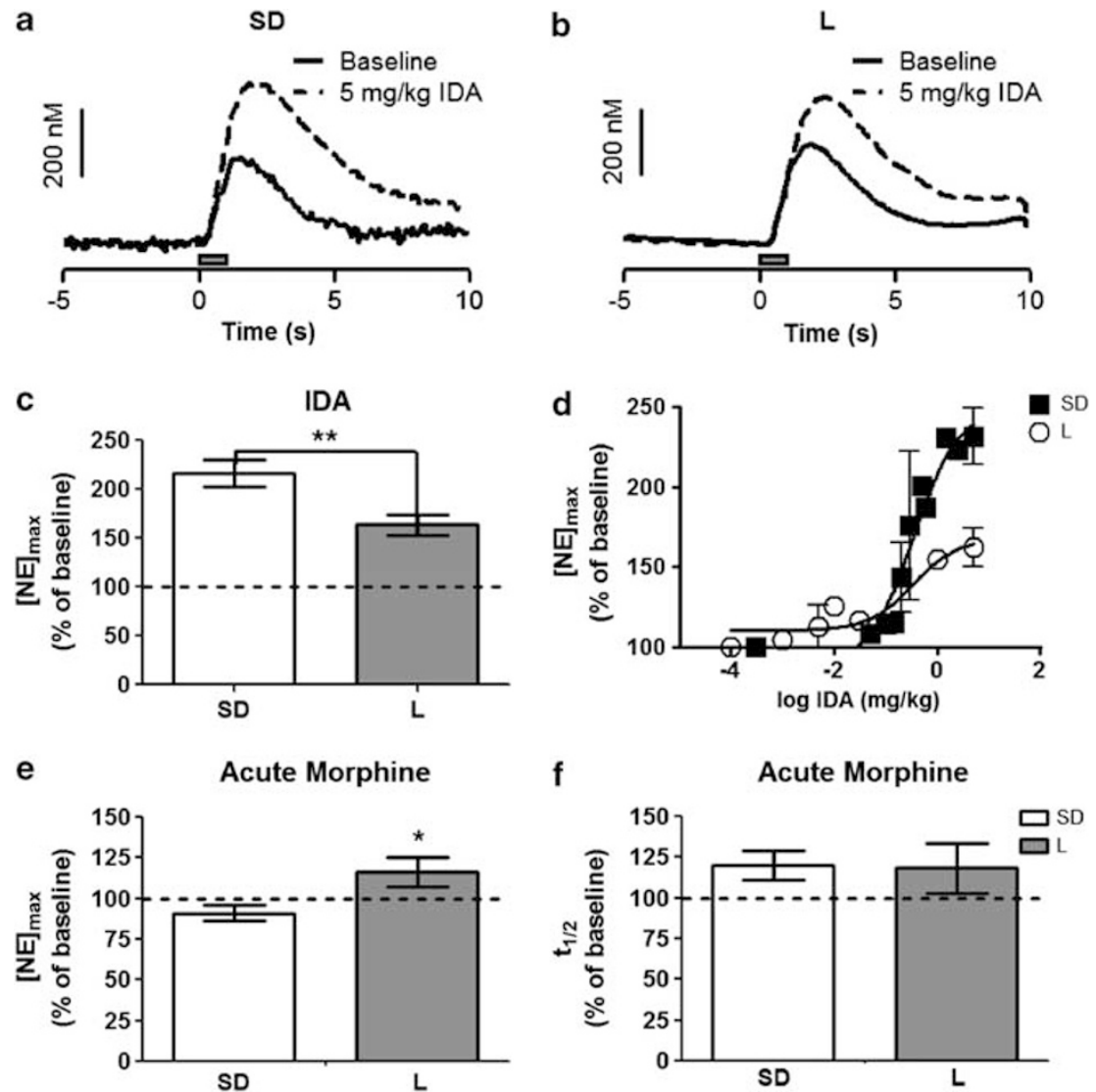

Figure 3 Effect of idazoxan (IDA) and morphine on norepinephrine release in the ventral bed nucleus of the stria terminalis (vBNST). ( $a$ and b) Representative concentration traces of Sprague-Dawley (SD) and Lewis ( $L$ ) rats, respectively. Solid line-evoked norepinephrine (NE) concentration with $60 \mathrm{~Hz}, 60$ pulses stimulation; dashed line - evoked NE concentration following $5 \mathrm{mg} / \mathrm{kg}$ IDA with the same electrical stimulus. (c) Average $[\mathrm{NE}]_{\max }$ following $5 \mathrm{mg} / \mathrm{kg}$ IDA relative to pre-drug stimulated release. (d) Change in $[\mathrm{NE}]_{\max }$ as percent of pre-drug baseline in both Sprague-Dawley (closed squares) and Lewis (open circles) rats, plotted vs log concentration of IDA ( $n=3$ each strain). (e) Average [NE] max to acute 5 mg/kg morphine relative to pre-drug stimulated release. ( $f$ ) Average clearance half-life following acute $5 \mathrm{mg} / \mathrm{kg}$ morphine relative to pre-drug stimulated release.

pre-drug baseline $v s$ L-SN $157 \pm 10 \%$ of pre-drug baseline, $n=5$; Figure $5 \mathrm{c}$ and d). Neither SD-MN, L-MN, nor L-SN groups were significantly different from the non-manipulated $\mathrm{L}$ group with respect to either $t_{1 / 2}$ or response to the IDA challenge. Similar to non-manipulated animals, we did not observe changes in $\alpha_{2}$-AR or NET binding in the vBNST of either strain or condition (SD-MN $n=5$, SD-SN $n=5$, L-MN $n=4$, L-SN $n=5$, Supplementary Figure 1 ).

\section{Catecholamine Tissue Content in Morphine-Dependent and Control Rats}

Finally, we compared the catecholamine tissue content in the BNST and NAc of MN animals and their controls (Table 1). Contrary to naïve animals, when NE tissue content was examined there was no significant interaction between MN and SN animals of each strain (2-way ANOVA, SD-MN: $1.97 \pm 0.49 \mu \mathrm{g} / \mathrm{g}, \quad n=9$; SD-SN $3.23 \pm 0.72 \mu \mathrm{g} / \mathrm{g}$, $n=8$; L-MN: $4.36 \pm 0.70 \mu \mathrm{g} / \mathrm{g}, n=5$; L-SN: $3.12 \pm 0.65 \mu \mathrm{g} / \mathrm{g}$, $n=5 \quad P>0.05)$. When BNST DA levels were examined, however, there was a main effect of both strain and drug treatment (2-way ANOVA, SD-MN: $1.32 \pm 0.22 \mu \mathrm{g} / \mathrm{g}, n=9$; SD-SN $0.71 \pm 0.17 \mu \mathrm{g} / \mathrm{g} n=8$; L-MN: $2.69 \pm 1.02 \mu \mathrm{g} / \mathrm{g}, n=5$; L-SN: $1.55 \pm 0.59 \mu \mathrm{g} / \mathrm{g}, \quad n=5, \quad P<0.05$ for each factor).
Interestingly, when DA levels in the NAc were measured, there was a significant interaction (2-way ANOVA, SD-MN: $5.21 \pm 0.91 \mu \mathrm{g} / \mathrm{g}, n=9$; SD-SN $4.85 \pm 0.75 \mu \mathrm{g} / \mathrm{g} n=8$; L-MN: $7.25 \pm 1.31 \mu \mathrm{g} / \mathrm{g}, \quad n=5 ; \quad \mathrm{L}-\mathrm{SN}: \quad 2.37+0.46 \mu \mathrm{g} / \mathrm{g}, \quad n=5$ $P<0.05)$. Post-hoc analysis revealed that L-MN rats had significantly higher DA in the NAc than their L-SN controls $(P<0.05)$, whereas SD animals showed no difference.

\section{DISCUSSION}

Previous studies have shown that $\mathrm{L}$ rats have anxiety and PTSD-like phenotypes (Cohen et al, 2006) and elevated extracellular NE in the BNST in response to a stressor (Pardon et al, 2002). They are also predisposed to substance abuse (Picetti et al, 2012; Sanchez-Cardoso et al, 2007). Here, we found that the tissue content of NE and DA in the vBNST, but not DA in the NAc, was elevated in L rats as compared with SD rats. Furthermore, using fast-scan cyclic voltammetry, we uncovered profound differences in the regulation of NE synaptic function in the vBNST in the two strains. NE is less regulated in $\mathrm{L}$ rats when compared with $\mathrm{SD}$ rats because they have a reduced NE uptake rate and less sensitivity to an $\alpha_{2}$-AR antagonist. When SD rats were made 


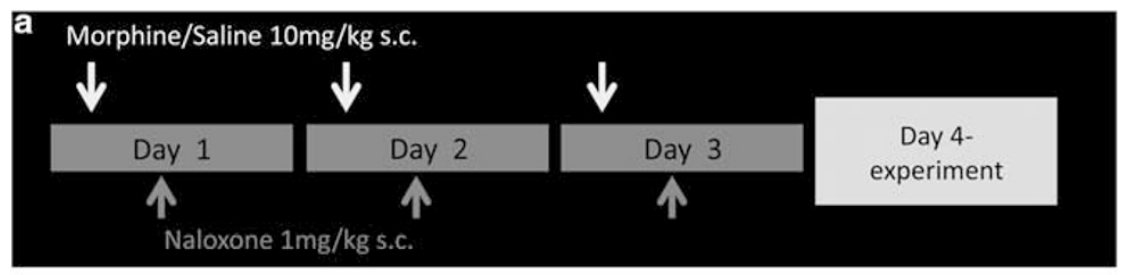

b

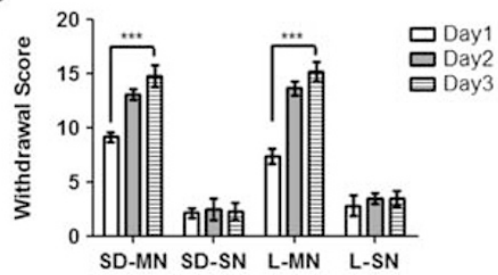

d
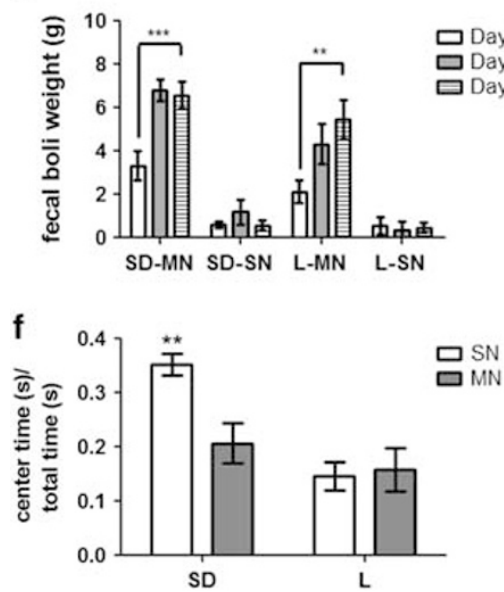

c

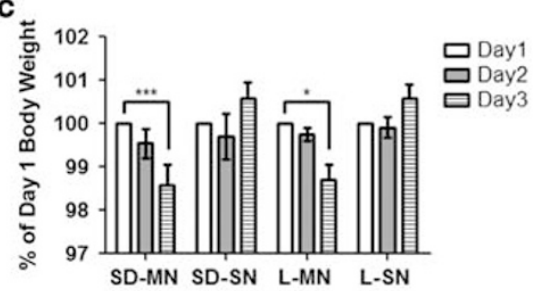

e
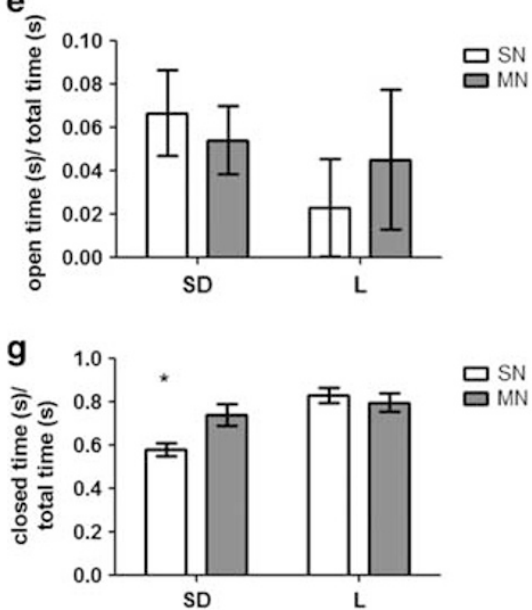

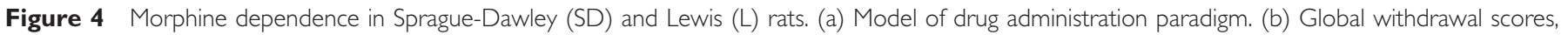

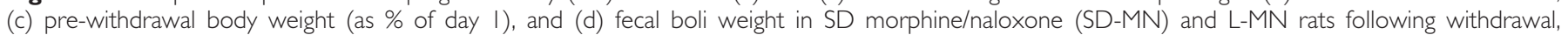

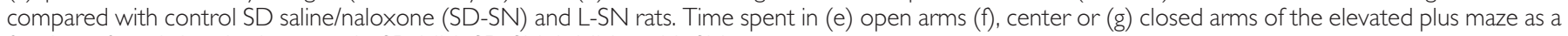
fraction of total time in the maze in SD-MN, SD-SN, L-MN, and L-SN rats.

dependent on morphine the two key regulators of $\mathrm{NE}$ overflow, uptake, and autoreceptor regulation, were both reduced such that $\mathrm{NE}$ neurotransmission resembled that of a non-manipulated $\mathrm{L}$ rat. In $\mathrm{L}$ rats, however, NE clearance and autoreceptor regulation were unaffected by morphine dependence. This physiological phenomenon correlated to an increase in anxiety-like behavior in SD-MN rats but not L-MN rats, as compared with their controls. The results demonstrate a robust plasticity within an organism's noradrenergic system that may be influenced by genetic factors as well as environmental insults.

\section{Rats Exhibited Poor Control Over NE Release and Clearance}

Neurotransmitter levels in the extracellular space are determined by the balance between release and uptake (Wightman et al, 1988). Release is a function of the rate of impulse flow (action potentials), the relative amount of $\mathrm{NE}$ in the releasable pool, and regulation by autoreceptors. The principal inhibitory autoreceptor in noradrenergic neurons is the $\alpha_{2}$-AR (Trendelenburg et al, 2001). NET is primarily responsible for NE clearance by uptake (Xu et al, 2000), and it follows Michaelis-Menten kinetics (Park et al, 2009). Electrical stimulation of NE neurons provides a convenient way to monitor release and uptake. Stimulation at supraphysiological frequencies allows release to predominate over uptake, enabling its nature to be examined. The maximum concentration achieved $\left([\mathrm{NE}]_{\max }\right)$, measured at the end of the stimulation, is thus predominantly a function of release. Recently the Jones lab has shown that the $t_{1 / 2}$ after the stimulation provides a measure of the rate of uptake that agrees with more complex Michaelis-Menten modeling (Yorgason et al, 2011). Thus, the electrical stimulations used here allowed quantification of several basic parameters that control extracellular NE.

Our studies showed that $[\mathrm{NE}]_{\max }$ is similar in the two strains with shorter stimulation trains. This is surprising because the vBNST NE content is twice as large in naïve $\mathrm{L}$ rats compared with SD rats. While release is linear in both strains, the slope of the regression line in the SD rats was significantly less than $\mathrm{L}$ rats, indicating that $\mathrm{L}$ rats have a larger releasable pool (Montague et al, 2004). Microdialysis studies have shown that extracellular NE increases during restraint stress in $\mathrm{L}$ rats relative to $\mathrm{SD}$ rats (Pardon et al, 2002), possibly arising from the larger releasable pool 
a

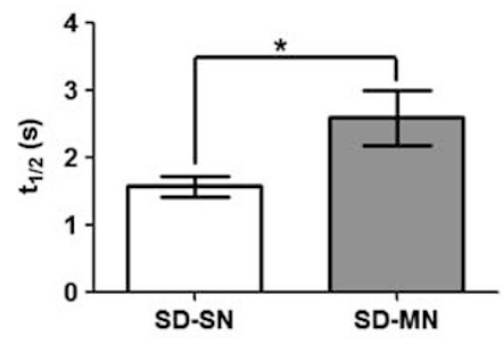

C

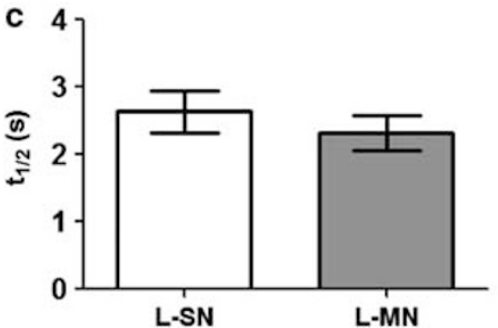

b
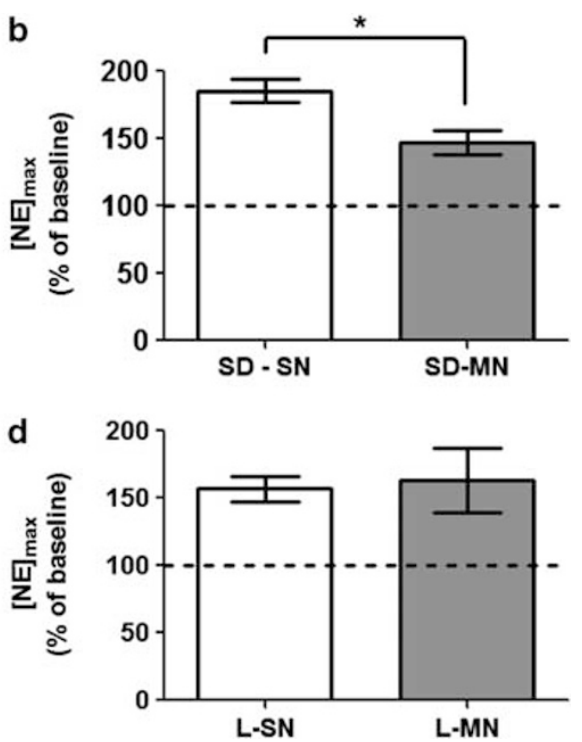

Figure 5 Clearance half-life and change in response following idazoxan (IDA) administration in morphine/naloxone (MN) animals vs saline controls. (a) $t_{1 / 2}$ and (b) change in $[N E]_{\max }$ as percent of pre-drug baseline in MN Sprague-Dawley (SD) rats vs saline controls $(P<0.05)$. (c) $t_{1 / 2}$ and $(d)$ change in $[\mathrm{NE}]_{\max }$ as percent of pre-drug baseline in MN Lewis (L) rats vs saline controls (n.s.). SN, saline/naloxone.

Table I Catecholamine Tissue Content Analysis $(\mu \mathrm{g} / \mathrm{g})$ in the Nucleus Accumbens and Ventral Bed Nucleus of the Stria Terminalis of Control (Saline/Naloxone) and Morphine-Dependent (Morphine/Naloxone) Rats by HPLC.

\begin{tabular}{lccc}
\hline & $\begin{array}{c}\text { DA NAc } \\
(\boldsymbol{\mu g} / \mathbf{g})\end{array}$ & $\begin{array}{c}\text { NE vBNST } \\
(\boldsymbol{\mu} \mathbf{g} / \mathbf{g})\end{array}$ & $\begin{array}{c}\text { DA vBNST } \\
(\boldsymbol{\mu} \mathbf{g} / \mathbf{g})\end{array}$ \\
\hline $\begin{array}{l}\text { Sprague-Dawley } \\
\text { Saline/naloxone }(n=8)\end{array}$ & $4.85 \pm 0.75$ & $3.23 \pm 0.72$ & $0.71 \pm 0.17$ \\
Morphine/naloxone $(n=9)$ & $5.21 \pm 0.91$ & $1.97 \pm 0.49$ & $1.32 \pm 0.22$ \\
& & & \\
Lewis & & & \\
Saline/naloxone $(n=5)$ & $2.37 \pm 0.46 *$ & $3.12 \pm 0.65$ & $1.23 \pm 0.37$ \\
Morphine/naloxone $(n=5)$ & $7.25 \pm 1.31 *$ & $4.36 \pm 0.70$ & $2.69 \pm 1.02$ \\
\hline
\end{tabular}

$* P<0.05$.

revealed in this work. Additionally, our experiments that probed the efficacy of the autoreceptor revealed that it exerts greater inhibition of release in SD rats. Although urethane anesthesia has been shown to stimulate $\alpha_{2}$-ARs in the periphery (Armstrong et al, 1982), our results in anesthetized animals mimicked the IDA effects observed in awake rats (Park et al, 2012). Taken together, our results suggest that $\mathrm{L}$ rats appear optimized for maximal $\mathrm{NE}$ neurotransmission. The combination of high content, low uptake rate, and low autoreceptor regulation ensures enhanced NE tone.

NE signaling in the BNST has been implicated in mediating control over the HPA axis and in anxiety-like behavior (Cecchi et al, 2002) and can release CRF within the BNST (McElligott et al, 2010; Nobis et al, 2011). Furthermore, prolonged NE signaling in the BNST induces synaptic plasticity that is modulated by stress (McElligott et al, 2010; McElligott and Winder, 2008). The altered mechanisms in
$\mathrm{L}$ rats that allow enhanced NE signaling may result in their anxiety-like phenotypes observed by others (Cohen et al, 2006), and could translate to anxiety-susceptible human populations. Indeed, recent data show that targeting noradrenergic signaling mechanisms may alleviate symptoms of substance abuse and PTSD (Raskind et al, 2000; Simpson et al, 2008).

\section{Morphine Dependence Enhanced Anxiety-Like Behavior, Increased NE Clearance Half-Life and Reduced Autoreceptor Function in SD but not L Rats}

It is hypothesized in the allostasis model that the progression to addiction requires the engagement of neuronal stress/anxiety systems, including the release of NE in the BNST (Delfs et al, 2000; Koob, 2009). Therefore, a predisposition to the negative emotional state associated with engagement of these neuronal substrates might explain the co-morbidity that is associated with anxiety disorders and addiction. L rats have a high anxiety/PTSD phenotype that is at least partially due to BNST function (Cohen et al, 2006; Duvarci et al, 2009) and a propensity to selfadminister and escalate opiate intake (Picetti et al, 2012; Sanchez-Cardoso et al, 2007). Interestingly, following withdrawal from morphine, but not acute morphine, SD rats showed profound plasticity in both autoreceptor regulation and uptake rates, as well as enhanced anxiety-like behavior. Moreover, the SD-MN rats had uptake rates and autoreceptor profiles indistinguishable from non-manipulated L rats. Surprisingly, L-MN rats exhibited neither this plasticity nor further increases in anxiety-like behavior, suggesting that autoreceptors and uptake rates in the $\mathrm{L}$ rats may be in a maximally depressed state. The correlation between vBNST $\mathrm{NE}$ and increased anxiety revealed in this work implicate plasticity at noradrenergic synapses as one mechanism for the development of anxiety and addiction phenotypes. 
Interestingly, our autoradiography experiments demonstrated that neither naïve, $\mathrm{MN}$, nor SN rats of either strain showed alterations in $\alpha_{2}$-AR or NET levels in the vBNST. This may be due to several reasons. First, this technique probes targets that are on the cell surface and in internal pools; therefore, it does not exclusively examine functional receptors and transporters. Second, it is possible that the functional changes we observed are not due to alterations in protein level, but due to a post-translational modification. Finally, the BNST has strong expression of nonspecific organic cation transporters, which also may have a role in the clearance of NE (Gasser et al, 2009) and could be manipulated by the gene-environment interactions we probed here. Nevertheless, the functional results clearly showed that the synaptic dynamics are dramatically different between SD and L rats, and that they were greatly altered in SD-MN rats.

Examining catecholamine tissue content in $\mathrm{MN}$ and control rats showed interesting changes in our treatment groups. Our data demonstrated that naloxone treatment lowered NE levels in the BNST in both strains regardless of pretreatment with morphine, and the effect was long lasting. These data are intriguing because naloxone and other $\mu$-opioid antagonists are prescribed to humans for opiate and alcohol dependence (Heilig and Egli, 2006). Further, we found that L-MN rats had elevated DA in their NAc as compared with their controls, which may also contribute to their self-administration escalation phenotype (Picetti et al, 2012).

We have shown that NE synapses in a genetic model of anxiety and addiction (L rats) permit exacerbated noradrenergic signaling due to reduced uptake and autoreceptor function. Furthermore, we showed that vBNST NE synapses in a relatively non-anxious $\mathrm{SD}$ rat resemble $\mathrm{L}$ rat $\mathrm{NE}$ synapses when SD rats are made morphine dependent. These data suggest that noradrenergic synapses undergo a plasticity event where increased NE signaling (here stemming from precipitated withdrawal) results in a biophysical/ biochemical change within the presynaptic cell to permit prolongation of future NE signaling on post synaptic cells. Furthermore, these alterations correlated to enhanced anxiety-like behavior in the SD rats. Additionally, the data support the allostasis model (Koob and Volkow, 2010) and the idea that genetic factors contributing to negative affect could in turn increase susceptibility to developing substance abuse issues.

\section{ACKNOWLEDGEMENTS}

We thank Dr Michael Saddoris for advice on statistical methods, Dr Ryan Vetrano and Dr Fulton Crews for behavioral advice and assistance, and Elyse Dankoski and Dr Thomas Kash for comments on an earlier version of the manuscript. This work was supported by an NIH grant (NS15841) to RMW and the NIMH Psychoactive Drug Screening Program Contract to BLR.

\section{DISCLOSURE}

BLR has received research support from Dai-Nippon Sumitomo and has been a consultant to Pfizer Pharmaceuticals, SeaChange Pharmaceuticals, Finnegan, Henderson,
Faraboug, Garnett and Henderson LLP and Dai-Nippon Sumitomo Pharmaceuticals. BLR also receives payment from the Journal of Clinical Investigation for his duties as Associate Editor of the JCI. The remaining authors declare no conflict of interest.

\section{REFERENCES}

Amitai N, Liu J, Schulteis G (2006). Discrete cues paired with naloxone-precipitated withdrawal from acute morphine dependence elicit conditioned withdrawal responses. Behav Pharmacol 17: 213-222.

Armstrong JM, Lefevre-Borg F, Scatton B, Cavero I (1982). Urethane inhibits cardiovascular responses mediated by the stimulation of alpha-2 adrenoceptors in the rat. J Pharmacol Exp Ther 223: 524-535.

Cecchi M, Khoshbouei H, Javors M, Morilak DA (2002). Modulatory effects of norepinephrine in the lateral bed nucleus of the stria terminalis on behavioral and neuroendocrine responses to acute stress. Neuroscience 112: 13-21.

Cohen H, Zohar J, Gidron Y, Matar MA, Belkind D, Loewenthal U et al (2006). Blunted HPA axis response to stress influences susceptibility to posttraumatic stress response in rats. Biol Psychiatry 59: 1208-1218.

Delfs JM, Zhu Y, Druhan JP, Aston-Jones G (2000). Noradrenaline in the ventral forebrain is critical for opiate withdrawal-induced aversion. Nature 403: 430-434.

Drolet G (2009). Progress in Neuro-Psychopharmacology and Biological Psychiatry. Elsevier Inc: Amsterdam.

Dumont EC, Williams JT (2004). Noradrenaline triggers GABAA inhibition of bed nucleus of the stria terminalis neurons projecting to the ventral tegmental area. J Neurosci 24: 8198-8204.

Duvarci S, Bauer EP, Pare D (2009). The bed nucleus of the stria terminalis mediates inter-individual variations in anxiety and fear. J Neurosci 29: 10357-10361.

Erb S, Hitchcott PK, Rajabi H, Mueller D, Shaham Y, Stewart J (2000). Alpha-2 adrenergic receptor agonists block stressinduced reinstatement of cocaine seeking. Neuropsychopharmacology 23: 138-150.

Forray MI, Gysling K (2004). Role of noradrenergic projections to the bed nucleus of the stria terminalis in the regulation of the hypothalamic-pituitary-adrenal axis. Brain Res Brain Res Rev 47: $145-160$.

Gasser PJ, Orchinik M, Raju I, Lowry CA (2009). Distribution of organic cation transporter 3, a corticosterone-sensitive monoamine transporter, in the rat brain. J Compar Neurol 512: 529-555.

George FR, Goldberg SR (1989). Genetic approaches to the analysis of addiction processes. Trends Pharmacol Sci 10: 78-83.

Heilig M, Egli M (2006). Pharmacological treatment of alcohol dependence: target symptoms and target mechanisms. Pharmacol Ther 111: 855-876.

Herr NR, Park J, McElligott ZA, Belle AM, Carelli RM, Wightman RM (2012). In vivo voltammetry monitoring of electrically evoked extracellular norepinephrine in subregions of the bed nucleus of the stria terminalis. J Neurophysiol 107: 1731-1737.

Hyman SM, Hong KI, Chaplin TM, Dabre Z, Comegys AD, Kimmerling A et al (2009). A stress-coping profile of opioid dependent individuals entering naltrexone treatment: a comparison with healthy controls. Psychol Addict Behav 23: 613-619.

John CE, Budygin EA, Mateo Y, Jones SR (2006). Neurochemical characterization of the release and uptake of dopamine in ventral tegmental area and serotonin in substantia nigra of the mouse. J Neurochem 96: 267-282.

Koob GF (2009). Brain stress systems in the amygdala and addiction. Brain Res 1293: 61-75. 
Koob GF, Volkow ND (2010). Neurocircuitry of addiction. Neuropsychopharmacology 35: 217-238.

Leri F, Flores J, Rodaros D, Stewart J (2002). Blockade of stressinduced but not cocaine-induced reinstatement by infusion of noradrenergic antagonists into the bed nucleus of the stria terminalis or the central nucleus of the amygdala. J Neurosci 22: 5713-5718.

McElligott ZA, Klug JR, Nobis WP, Patel S, Grueter BA, Kash TL et al (2010). Distinct forms of Gq-receptor-dependent plasticity of excitatory transmission in the BNST are differentially affected by stress. Proc Natl Acad Sci USA 107: 2271-2276.

McElligott ZA, Winder DG (2008). Alpha1-adrenergic receptorinduced heterosynaptic long-term depression in the bed nucleus of the stria terminalis is disrupted in mouse models of affective disorders. Neuropsychopharmacology 33: 2313-2323.

McElligott ZA, Winder DG (2009). Modulation of glutamatergic synaptic transmission in the bed nucleus of the stria terminalis. Prog Neuropsychopharmacol Biol Psychiatry 33: 1329-1335.

Meloni EG, Gerety LP, Knoll AT, Cohen BM, Carlezon Jr WA (2006). Behavioral and anatomical interactions between dopamine and corticotropin-releasing factor in the rat. J Neurosci 26: 3855-3863.

Montague PR, McClure SM, Baldwin PR, Phillips PE, Budygin EA, Stuber GD et al (2004). Dynamic gain control of dopamine delivery in freely moving animals. J Neurosci 24: 1754-1759.

Nobis WP, Kash TL, Silberman Y, Winder DG (2011). betaAdrenergic receptors enhance excitatory transmission in the bed nucleus of the stria terminalis through a corticotrophinreleasing factor receptor-dependent and cocaine-regulated mechanism. Biological psychiatry 69: 1083-1090.

Pardon MC, Gould GG, Garcia A, Phillips L, Cook MC, Miller SA et al (2002). Stress reactivity of the brain noradrenergic system in three rat strains differing in their neuroendocrine and behavioral responses to stress: implications for susceptibility to stress-related neuropsychiatric disorders. Neuroscience 115: 229-242.

Park J, Kile BM, Wightman RM (2009). In vivo voltammetric monitoring of norepinephrine release in the rat ventral bed nucleus of the stria terminalis and anteroventral thalamic nucleus. Eur J Neurosci 30: 2121-2133.

Park J, Wheeler RA, Fontillas K, Keithley RB, Carelli RM, Wightman RM (2012). Catecholamines in the bed nucleus of the stria terminalis reciprocally respond to reward and aversion. Biol Psychiatry 71: 327-334.
Picetti R, Caccavo JA, Ho A, Kreek MJ (2012). Dose escalation and dose preference in extended-access heroin self-administration in Lewis and Fischer rats. Psychopharmacology 220: 163-172.

Raskind MA, Dobie DJ, Kanter ED, Petrie EC, Thompson CE, Peskind ER (2000). The alpha1-adrenergic antagonist prazosin ameliorates combat trauma nightmares in veterans with posttraumatic stress disorder: a report of 4 cases. J Clin Psychiatry 61: $129-133$.

Sanchez-Cardoso P, Higuera-Matas A, Martin S, del Olmo N, Miguens M, Garcia-Lecumberri C et al (2007). Modulation of the endogenous opioid system after morphine self-administration and during its extinction: a study in Lewis and Fischer 344 rats. Neuropharmacology 52: 931-948.

Schulteis G, Heyser CJ, Koob GF (1999). Differential expression of response-disruptive and somatic indices of opiate withdrawal during the initiation and development of opiate dependence. Behav Pharmacol 10: 235-242.

Simpson TL, Saxon AJ, Meredith CW, Malte CA, McBride B, Ferguson LC et al (2008). A pilot trial of the alpha-1 adrenergic antagonist, Prazosin, for alcohol dependence. Alcohol Clin Exp Res 33: 255-263.

Sinha R (2008). Chronic stress, drug use, and vulnerability to addiction. Ann N Y Acad Sci 1141: 105-130.

Trendelenburg AU, Klebroff W, Hein L, Starke K (2001). A study of presynaptic alpha2-autoreceptors in alpha2A/D-, alpha2B- and alpha2C-adrenoceptor-deficient mice. Naunyn-Schmiedeberg Arch Pharmacol 364: 117-130.

Trescot AM, Datta S, Lee M, Hansen H (2008). Opioid pharmacology. Pain Physician 11(2 Suppl): S133-S153.

Wang X, Cen X, Lu L (2001). Noradrenaline in the bed nucleus of the stria terminalis is critical for stress-induced reactivation of morphine-conditioned place preference in rats. Eur J Pharmacol 432: $153-161$.

Wightman RM, Amatore C, Engstrom RC, Hale PD, Kristensen EW, Kuhr WG et al (1988). Real-time characterization of dopamine overflow and uptake in the rat striatum. Neuroscience 25: 513-523.

Xu F, Gainetdinov RR, Wetsel WC, Jones SR, Bohn LM, Miller GW et al (2000). Mice lacking the norepinephrine transporter are supersensitive to psychostimulants. Nat Neurosci 3: 465-471.

Yorgason JT, Espana RA, Jones SR (2011). Demon voltammetry and analysis software: analysis of cocaine-induced alterations in dopamine signaling using multiple kinetic measures. J Neurosci Methods 202: 158-164.

Supplementary Information accompanies the paper on the Neuropsychopharmacology website (http://www.nature.com/npp) 